\title{
Overall survival and prognostic factors in diabetic patients with invasive fungal rhinosinusitis
}

\author{
Thwe Phyo Kan Nyunt, ${ }^{1,2}$ Baharudin Abdullah, ${ }^{3}$ Maung Maung Khaing, ${ }^{4}$ Kachorn Seresirikachorn, ${ }^{1,2}$ Norasnieda Md Shukri, ${ }^{3}$ \\ Songklot Aeumjaturapat, ${ }^{1,2}$ Supinda Chusakul, ${ }^{1,2}$ Jesada Kanjanaumporn, ${ }^{1,2}$ Richard J Harvey, ${ }^{5,6}$ Kornkiat Snidvongs ${ }^{1,2}$
}

\begin{abstract}
Background: Patients with diabetes mellitus (DM) are susceptible to invasive fungal rhinosinusitis (IFRS). The mortality rate of IFRS varies greatly among the patients with DM.
\end{abstract}

Objective: To identify the prognostic factors for the overall survival of patients with DM and IFRS.

Methods: A retrospective study was conducted in four tertiary hospitals in Thailand, Malaysia and Myanmar. Patients diagnosed with IFRS and DM from 2008 to 2019 were identified. The outcome was the overall survival. Variables analyzed for risk factors were age, HbA1C level, ketoacidosis, white blood cell count, hyperglycemia, duration of DM, current use of diabetic medications, serum creatinine level, and the extensions of IFRS to the orbit, the cavernous sinus and intracranial cavity.

Results: Sixty-five diabetic patients with IFRS (age $57.9 \pm 13.4$ years, male $60 \%$ ) were identified. The mortality rate was $21.5 \%$. The extensions of IFRS to the cavernous sinus (hazard ratio 5.1, 95\% CI [1.4-18.2], $p=0.01$ ) and intracranial cavity (hazard ratio 3.4, 95\% CI [1.1-11.3, $p=0.05$ ) predicted mortality. Current use of diabetic medications decreased the mortality risk (hazard ratio $0.2,95 \%$ CI [0.1-0.9], $p=0.03$ ). The 6-month overall survival of the patients with and without the cavernous sinus extension were $51.4 \%$ and $83.6 \%,(p=0.001)$, with and without intracranial extension $53.3 \%$ and $88.9 \%,(p=0.001)$, and with and without current diabetic medications $82.3 \%$ and $57.5 \%$, respectively $(p=$ 0.045).

Conclusion: The extensions of IFRS to the cavernous sinus and intracranial cavity increased the risk of death in patients with DM. Survival was primarily related to current use of diabetic medications.

Key words: diabetes mellitus, fungal, rhinosinusitis, risk factors, cavernous sinus

\footnotetext{
From:

Department of Otolaryngology, Faculty of Medicine, Chulalongkorn University, Bangkok, Thailand

2 Endoscopic Nasal and Sinus Surgery Excellence Center, King Chulalongkorn Memorial Hospital, Bangkok, Thailand

3 Department of Otorhinolaryngology-Head \& Neck Surgery, School of Medical Sciences, Universiti Sains Malaysia, Kubang Kerian, Kelantan, Malaysia

${ }^{4}$ Department of Otorhinolaryngology-Head \& Neck Surgery, University of Medicine (1), Yangon, Myanmar

Faculty of Medicine, Health and Human Sciences, Macquarie University, Sydney, Australia

${ }^{6}$ Rhinology and Skull Base Research group, Applied Medical Research Centre, University of New South Wales, Sydney, Australia
}

\section{Corresponding author:}

Kornkiat Snidvongs

Faculty of Medicine, Chulalongkorn University, Pathumwan,

Bangkok10330, Thailand

E-mail: drkornkiat@yahoo.com

\section{Introduction}

Patients with poorly controlled diabetes mellitus (DM) are acknowledged to be immunocompromised and susceptible to infections. Hyperglycemia activates protein kinase $\mathrm{C}$ and inhibits the production of polymorphonuclear cells, neutrophil migration, and the formation of neutrophil extracellular traps. Chemotaxis and phagocytic activity become ineffective. The expression of class I major histocompatibility complex is diminished. ${ }^{1}$ In addition, the structure of complement is altered with an imbalance between the complement activation and restriction. $^{2,3}$

Aspergillosis and mucormycosis can cause invasive fungal rhinosinusitis (IFRS) in immunocompromised hosts. ${ }^{4,5}$ Spore germination and tissue invasion cause severe endothelial damage, angioinvasion, and extensive necrosis. Initial presenting symptoms include fever, facial pain, colored nasal discharge and orbital symptoms. Rapid progression into nearby 
structures is characteristic of this disease. The extensions to the orbit, intracranial cavity and the cavernous sinus are the most common complications. ${ }^{6}$ The morbidity rate of IFRS is quite high, around $20 \%$ to $50 \% .^{6} \mathrm{DM}$ is acknowledged as an underlying disease related to mortality. However, the survival rate of IFRS in patients with DM is greater than in patients with another underlying immunocompromised disease, hematologic malignancy. ${ }^{7}$ The immune status of diabetic patients should be a key factor which predicts the overall survival of diabetic patients with IFRS. If the impaired immune status can be recovered, favorable therapeutic outcomes can be achieved. This study aims to identify key prognostic factors for the overall survival of DM patients with IFRS.

\section{Material and Methods}

A retrospective study was conducted in three countries in Southeast Asia (Thailand, Malaysia and Myanmar). Medical records from 1 January 2008 to 31 December 2019 of four tertiary University hospitals were reviewed. This study was approved by the Institutional Review Board of all four hospitals, including (1) The King Chulalongkorn Memorial Hospital, Bangkok, Thailand (IRB number 085/62), (2) Ear, Nose, Throat \& Head and Neck Surgery Hospital, Yangon, Myanmar (IRB number 184 (ENT)/UMM/2018), (3) Eye, Ear, nose, Throat \& Head and Neck surgery hospital, Mandalay, Myanmar (IRB number 184 (ENT)/UMM/2018), and (4) Hospital of Universiti Sains Malaysia (IRB number USM/ JEPeM/19030215). This research was granted by “The $90^{\text {th }}$ Anniversary of Chulalongkorn University Scholarship". Patients diagnosed with IFRS and DM at any age were identified. The diagnosis criteria of IFRS and DM were according to the ICD-10-CM codes. Patients with DM either previously diagnosed or newly diagnosed at the time of admission were included. Patients with incomplete records were excluded from the study. Diagnostic criteria for DM were a fasting plasma glucose value $\geq 7.0 \mathrm{mmol} / \mathrm{L}(126 \mathrm{mg} / \mathrm{dl})$, a 2 -hour post-load plasma glucose $\geq 11.1 \mathrm{mmol} / \mathrm{L}(200 \mathrm{mg} / \mathrm{dl})$, a HbA1C level $\geq 6.5 \%(48 \mathrm{mmol} / \mathrm{mol})$, or a random plasma glucose $\geq 11.1$ $\mathrm{mmol} / \mathrm{L}(200 \mathrm{mg} / \mathrm{dl})$ with the presence of signs and symptoms. ${ }^{8,9}$ Diagnostic criteria for IFRS were radiological imaging and/or histopathological evidence of hyphal forms within the sinus mucosa, submucosa, blood vessels, or bone. ${ }^{10}$

The primary outcome of this study was the overall survival. Secondary outcomes were prognostic factors for the overall survival. Duration from the admission date to either the last follow-up date or death was recorded. Variables were analyzed for prognostic factors which included: old age, high $\mathrm{HbA1C}$ level, ketoacidosis, white blood cell count, hyperglycemia, duration of DM, current use of diabetic medications, serum creatinine level, the extensions of IFRS to the orbit, the cavernous sinus and intracranial cavity. Old age was defined as 60 years old. ${ }^{11}$ High HbA1C level was defined as a level above 8. ${ }^{12}$ Diabetic ketoacidosis was diagnosed when the patients had hyperglycemia $>250 \mathrm{mg} / \mathrm{dl}$, a presence of either serum or urine ketone and an arterial $\mathrm{pH}<7.3 .^{13}$ Leukopenia was defined as a white blood cell count $<4,000 \times 10^{3}$ cell per liter, ${ }^{14}$ and leukocytosis $>11,000 \times 10^{3}$ cell per liter. ${ }^{15}$ Hyperglycemia was defined as a plasma glucose level on the admission day above $200 \mathrm{mg} / \mathrm{dl} .^{12}$ Current use of diabetic medications was recorded as yes or no. A serum creatinine level greater than 1 $\mathrm{mg} / \mathrm{dl}$ was defined as high. The orbital extension, the cavernous sinus extension and intracranial extension of IFRS were recorded as yes or no.

\section{Statistical analysis}

Descriptive data were presented as percentage and mean \pm standard deviation. Univariate analysis was done for each variable. Significant variables were incorporated to a multivariate model. Backward stepwise Cox proportional hazard model was run to assess potential hazard ratio. Kaplan Meier curve and Log-rank test were used for analyzing survival outcomes. STATA 15 was used for the data analysis. A $p$-value $\leq$ 0.05 was considered statistically significant.

\section{Results \\ Patients}

A total of 65 diabetic patients with IFRS were identified. The mean age was $57.9 \pm 13.4$ years and $60 \%$ were male. Duration of the admission date to the last follow-up date was $207 \pm 161$ days. Type II diabetes accounted for $98.4 \%$ of the patients and 16 patients $(24.6 \%)$ were newly diagnosed with type II diabetes at the time of admission. All previously diagnosed diabetic patients were taking medications for controlling their plasma glucose level. Newly diagnosed diabetic patients had a mortality rate of $31.2 \%$ while known diabetic patients had $18.4 \%$. Four patients had retinopathy and six patients had nephropathy. None had neuropathy. Seven patients (10.8\%) had diabetic ketoacidosis. Three of 7 patients were newly diagnosed.

Mucormycosis was diagnosed in $35.4 \%$ and aspergillosis in $35.4 \%$ of the patients. Seven patients (10.8\%) had both mucormycosis and aspergillosis. One patient (1.5\%) had both mucormycosis and actinomycosis. Other types of fungi such as Candida species accounted for $1.5 \%$. The type of fungal hyphae was not specified in $6.2 \%$. The culture results that had sterile fungal culture were reported in 6 patients $(9.2 \%)$. Fifty-six patients $(86.2 \%)$ had acute IFRS and 9 patients $(13.8 \%)$ had chronic IFRS. The primary sites of IFRS involvement were maxillary sinus (54 patients, $83 \%$ ), ethmoid sinus (38 patients, $58 \%$ ), sphenoid sinus (23 patients, 35\%), frontal sinus (2 patients, $3 \%$ ), and orbit (3 patients, 5\%).

Sixty-two patients $(95.4 \%)$ received antifungal treatment and three patients did not received antifungal treatment. Forty-two patients $(64.6 \%)$ received amphotericin B derivatives therapy and 11 patients received amphotericin B derivatives as a sole agent. Twenty-nine patients (44.6\%) received voriconazole. All patients underwent endoscopic sinus surgery. Thirty-three patients $(50.8 \%)$ underwent one endoscopic sinus surgery. Seventeen patients $(26.1 \%)$ and fifteen patients (23.1\%) underwent two surgeries and more than two surgeries, respectively. Sinus surgery was combined with orbital surgery in 16 patients $(24.6 \%)$ and neurosurgery in 3 patients (4.6\%). 8 (24.2\%) patients out of 33 patients who received surgery for only one time died in this study. Where 2 patients out of 17 patients died in 2 times surgeries and 4 patients out of 12 patients died in 3 times surgeries. 


\section{Prognostic factors and overall survival analysis}

The overall survival analysis was obtained from the data of 65 patients. The mortality rate was $21.5 \%$. All fatal cases were acute IFRS. Nine patients (100\%) with chronic IFRS survived. Eight out of 33 patients (24.2\%) who received one endoscopic sinus surgery and six out of 32 patients $(18.8 \%)$ who received multiple endoscopic sinus surgeries died. The mean follow-up duration was 207.5 \pm 161.9 days (range 2-365 days). Thirty-one patients $(47.7 \%)$ were over 60 years old. Plasma glucose levels were reported in all the 65 patients. The mean plasma glucose level was $220.1 \pm 98.7 \mathrm{mg} / \mathrm{dl}$ and $30 \mathrm{pa}-$ tients (46.2\%) had plasma glucose above $200 \mathrm{mg} / \mathrm{dl}$. HbAlC level was reported in 39 patients. The mean $\mathrm{HbA1C}$ level was $10.6 \pm 3.5$ and 31 patients had HbA1C greater than 8 . Duration of having diabetes was recorded in 51 patients. The mean duration of having diabetes before admission was $7.2 \pm 8.5$ years. Seventeen patients $(33.3 \%)$ had less than one-year duration, 17 patients $(33.3 \%)$ between $1-10$ years, and 17 patients (33.3\%) above ten years. Sixteen patients (24.6\%) did not take any diabetic medication at the time of admission. Total white blood cell count was recorded in 62 patients. The mean total white blood cell count was 14,572.3 $\pm 24,670.3 \times 10^{3}$ cell per liter. Two patients $(3.2 \%)$ had a total white blood cell count less than $4,000 \times 10^{3}$ cell per liter, 35 patients $(56.5 \%)$ had $4,000-11,000 \times 10^{3}$ cell per liter, and 25 patients $(40.3 \%)$ had greater than $11,000 \times 10^{3}$ cell per liter. Serum creatinine level was recorded in 63 patients. The mean serum creatinine level was $1.2 \pm 0.9 \mathrm{mg} / \mathrm{dl}$. Thirty-five patients (55.6\%) had serum creatinine level greater than $1 \mathrm{mg} / \mathrm{dl}$. Seven patients $(10.8 \%)$ had diabetic ketoacidosis. All patients had imaging records and 15 patients $(23.1 \%)$ had the cavernous sinus extension, 23 patients $(35.4 \%)$ had intracranial extension and 37 patients (56.9\%) had the orbital extension. Among these patients with extensive lesion, there were 6 patients who had overlapping of intracranial, cavernous sinus, orbital extension. Out of these 6 patients, five $(83.3 \%)$ patients died within 6 months.

Univariable logistic regression analysis revealed three statistically significant risk factors: the orbital extension (hazard ratio $4.7,95 \%$ CI $[1.1-21.2], p=0.004)$, the cavernous sinus extension (hazard ratio $4.7,95 \%$ CI [1.6-13.4], $p=0.004$ ), and intracranial extension (hazard ratio 5.7, 95\% CI [1.8-18.1], $p$ $=0.03)$. Current use of diabetic medications was a protective factor (hazard ratio $0.4,95 \%$ CI $[0.12-1.0], p=0.05$ ). Multivariable logistic regression analysis confirmed two independent risk factors: the cavernous sinus extension (hazard ratio 5.1, 95\% CI [1.4-18.2], $p=0.013$ ) and intracranial extension

Table 1. Prognostic factors for overall survival of invasive fungal rhinosinusitis in diabetic patients

\begin{tabular}{|c|c|c|c|c|c|c|c|c|}
\hline \multirow[b]{3}{*}{ Orbital extension } & \multicolumn{4}{|c|}{ Univariate analysis } & \multicolumn{4}{|c|}{ Multivariate analysis } \\
\hline & \multirow{2}{*}{$\begin{array}{c}\begin{array}{c}\text { Hazard } \\
\text { ratio }\end{array} \\
4.7\end{array}$} & \multicolumn{2}{|c|}{$\begin{array}{l}\text { 95\% Confidence } \\
\text { Interval }\end{array}$} & \multirow{2}{*}{$\begin{array}{c}\boldsymbol{p} \text {-value } \\
0.042\end{array}$} & \multirow{2}{*}{$\begin{array}{c}\begin{array}{c}\text { Hazard } \\
\text { ratio }\end{array} \\
2.7\end{array}$} & \multicolumn{2}{|c|}{$\begin{array}{l}\text { 95\% Confidence } \\
\text { Interval }\end{array}$} & \multirow{2}{*}{$\begin{array}{c}\boldsymbol{p} \text {-value } \\
0.204\end{array}$} \\
\hline & & 1.1 & 21.2 & & & 0.6 & 12.4 & \\
\hline Cavernous sinus extension & 4.7 & 1.6 & 13.4 & 0.004 & 5.1 & 1.4 & 18.2 & 0.013 \\
\hline Intracranial extension & 5.7 & 1.8 & 18.1 & 0.03 & 3.4 & 1.0 & 11.3 & 0.046 \\
\hline Current use of diabetic medications & 0.4 & 0.1 & 1.0 & 0.05 & 0.2 & 0.1 & 0.9 & 0.028 \\
\hline Age $>60$ & 1.5 & 0.5 & 4.5 & 0.42 & & & & \\
\hline Duration of $\mathrm{DM}>1$ year & 0.6 & 0.3 & 1.3 & 0.17 & & & & \\
\hline Plasma glucose $>200 \mathrm{mg} / \mathrm{dl}$ & 1.5 & 0.5 & 4.4 & 0.44 & & & & \\
\hline $\mathrm{HbA} 1 \mathrm{C}>8$ & 1.8 & 0.2 & 15.7 & 0.55 & & & & \\
\hline Total WBC count & 1.8 & 0.6 & 5.1 & 0.26 & & & & \\
\hline Serum creatinine level $>1 \mathrm{mg} / \mathrm{dl}$ & 1.2 & 0.4 & 3.5 & 0.73 & & & & \\
\hline
\end{tabular}

Abbreviations: $\mathrm{DM}=$ diabetes mellitus, $\mathrm{WBC}=$ white blood cell

Table 2. Overall survival at 1, 3 and 6 months of invasive fungal rhinosinusitis in diabetic patients who had risk factors compared to those without

\begin{tabular}{|lcccc|}
\hline \multicolumn{1}{|c}{ Overall survival (\%) } & 1 month & $\mathbf{3}$ months & $\mathbf{6}$ months & $\boldsymbol{p}$-value \\
\hline Intracranial extension & 65.2 & 60.2 & 53.3 & 0.001 \\
\hline No intracranial extension & 97.3 & 88.9 & 88.9 & \\
\hline Cavernous sinus extension & 60.0 & 51.4 & 51.4 & 0.001 \\
\hline No cavernous sinus extension & 93.3 & 86.4 & 83.6 & \\
\hline Current no use of diabetic medications & 74.5 & 67.0 & 57.5 & 0.045 \\
\hline Current use of diabetic medications & 89.3 & 82.3 & 82.3 & \\
\hline
\end{tabular}




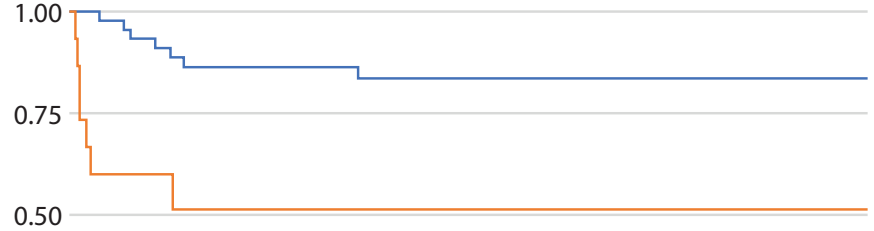

0.25

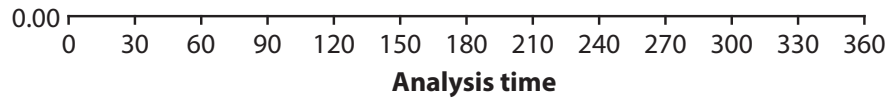

- No Cavernous Sinus Extension Cavernous Sinus Extension Significance was assessed by Log rank test. $(P=0.0016)$

Figure 1A. Kaplan Meier Estimates of Overall Survival for the Cavernous Sinus Extension.

Cav Sinus $=$ cavernous sinus

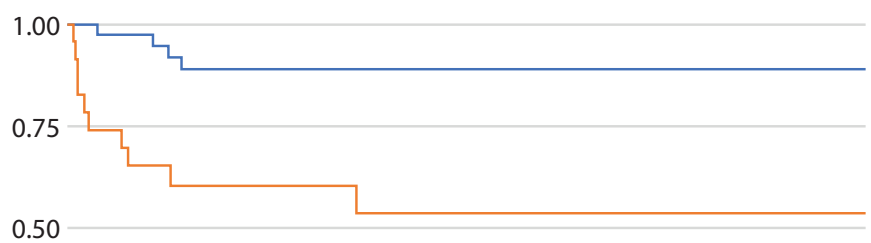

0.25

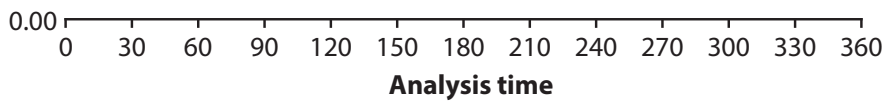

- No Intracranial Extension - Intracranial Extension

Significance was assessed by Log rank test. $(P=0.0010)$

Figure 1B. Kaplan Meier Estimates of Survival for Intracranial Extension.

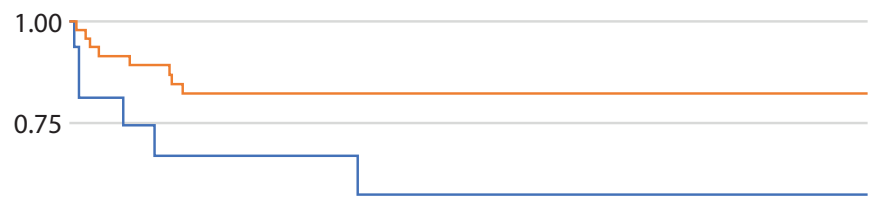

0.50

0.25

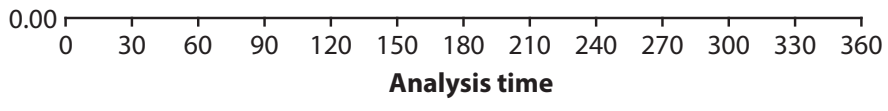

- Current no use of Diabetic Medications

- Current use of Diabetic Medications

Significance was assessed by Log rank test. $(P=0.0455)$

Figure 1C. Kaplan Meier Estimates of Overall Survival for Current use of Diabetic Medications. (hazard ratio 3.4, 95\% CI [1.0-11.3], $p=0.046$ ). Current use of diabetic medications was an independent protective factor (hazard ratio $0.2,95 \%$ CI $[0.1-0.9], p=0.03$ ). The data are displayed in Table 1. The 6-month overall survival of the patients with the cavernous sinus extension was $51.4 \%$ compared to $83.6 \%$ in the patients without the cavernous sinus extension $(p=0.001)$. See Table 2 and Figure 1A. The 6-month overall survival of the patients with intracranial extension was $53.3 \%$ compared to $88.9 \%$ in the patients without intracranial extension $(p=0.001)$. See Figure 1B. The 6-month overall survival of the patients who did not take any diabetic medication was $57.5 \%$ compared to $82.3 \%$ in the patients who took medications $(p=0.045)$. See Figure 1C.

\section{Discussion}

This study found that the overall survival of diabetic patients with IFRS was greatly diminished when the IFRS extension involved the cavernous sinus and intracranial cavity. Angioinvasion is a hallmark of IFRS. The resting spores of the fungi that swell and germinate within a host cause rapid filamentous growth, angioinvasion, and vessel thrombosis. ${ }^{16}$ Tissue invasion and angioinvasion are rapid and progressive which lead to extension of the IFRS into the cavernous sinus. Common clinical manifestations of cavernous sinus involvement are spiking fevers together with bilateral orbital signs including bilateral exophthalmos, lid drop, and complete ophthalmoplegia. ${ }^{17}$ Signs of meningeal irritation are also common in IFRS with the cavernous sinus extension. IFRS with the cavernous sinus extension is acknowledged as a fatal condition. The areas of both the cavernous sinus and intracranial extensions are high risk for endoscopic sinus and skull base debridement. As a result, total disease eradication of these areas may not be achieved. The findings of this study did not show that multiple endoscopic sinus surgeries reduced the risk.

There were 37 patients had orbital extension of the IFRS, including orbital apex syndrome and optic nerve invasion. Out of 37, twelve patients (32\%) died. We found that the prognosis was not bad because orbital exenteration was the factor bringing good results. Although the orbital extension was a significant risk factor when it was assessed by univariable logistic regression, it was no longer statistically significant by multivariable logistic regression. Therefore, the orbital extension was not an independent factor. It was significant due to its association with the cavernous sinus extension. For a total disease eradication of IFRS extension to the orbit, an orbital exenteration is suggested when significant amount of the orbital contents are invaded. Nevertheless, the orbit can be preserved in selected cases with minimal orbital involvement. In this study the authors did not acknowledge blindness from an orbital exenteration as a morbidity of IFRS because the orbital exenteration was for saving the patient's life. Besides, patients with blindness may function normally and have a good quality of life. 
Current use of diabetic medications was a protective factor. The immunocompromised status of diabetic patients is related to the diabetes status. Patients with poorly controlled DM have impaired immune function. Both humoral and cellular innate immunity of the patients with diabetes are defective. Glycation end products play a causative role in the vascular complications of DM and the decreased expression of class I major histocompatibility complex on the surface of myeloid cells. The interleukin (IL)-10 production by myeloid cells, the interferon (IFN)- $\gamma$ and tumor necrosis factor (TNF)- $\alpha$ productions by $\mathrm{T}$ cells, and the secretions of IL- 1 and IL- 6 by mononuclear cells and monocytes are inhibited. ${ }^{1}$ The balance between complement activation and restriction is broken. As a result, poorly controlled DM activates protein kinase $\mathrm{C}$ which inhibits neutrophil migration, decreases the production of polymorphonuclear cells, decreases chemotaxis and decreases phagocytic activity. The mortality rate of IFRS has a wide range from $20 \%$ to $80 \%$ and depends on the diabetes status. Well-controlled DM by appropriate medications can recover the impaired immune function. However, the analysis of plasma glucose and HbA1C levels in this study did not reach statistical significance. The cut-off points of $200 \mathrm{mg} / \mathrm{dl}$ used for analyzing plasma glucose and the cut-off point of 8 for $\mathrm{HbA1C}$ may not be sensitive. There was missing data for HbA1C which caused non-significant results. Post-hoc ROC was performed to define a cut-off point for HbA1C. The cutoff point of $\mathrm{HbA1C}$ was 9.2 which gave sensitivity of 0.86 and specificity of 0.80 still did not reach statistical significance.

In line with the findings of our study, a retrospective study by Sun, et al. ${ }^{18}$ showed that the extension of the fungal tissue invasion was a risk factor associated with the mortality rate. Data from 13 patients with IFRS from their cohort and 77 patients in the literature were assessed. Fifty-seven percent of the patients had intracranial involvement with a $74 \%$ mortality rate. This mortality rate was higher than the overall mortality rate $(52 \%)$ of the patients. Likewise, a retrospective study by Jung, et al. ${ }^{19}$ assessed 12 patients with rhinocerebral mucormycosis. The most common underlying immunocompromised diseases were DM and hematological malignancies. The overall mortality rate was $33 \%$. All the fatal cases were DM. The mortality was related to uncontrolled underlying disease. The risk factor was the extension of disease to the orbit or intracranial cavity. ${ }^{19}$

This study had several limitations. First, this was a retrospective study. Medical records retrieval went back to January 2008 which was before electronic medical records were used in the hospitals. The data extraction was from a combination of electronic and hard copies. There were missing variables or incomplete data. The patients with missing variables or incomplete data were excluded from the variable analysis. Therefore, the total number of the patients assessed for each variable was different. The small number of the patients in some variables may not have enough power to show any significance. However, the statistically significant variables showed from these small numbers were accurate and true because the authors did not make assumption on the missing data. Another limitation was the diagnostic criteria used for DM and IFRS may vary across the institutes. Nevertheless, this study followed the ICD-10-CM that have been generally accepted. Records with inappropriate diagnosis or unclear data were excluded from the study.

\section{Conclusions}

The overall mortality rate of diabetic patients with IFRS in this study was $21.5 \%$. The extensions of IFRS to the cavernous sinus and intracranial cavity were significant risk factors which predicted death. Survival was primarily related to current use of diabetic medications. In practice, treatment should aim for controlling diabetic conditions and a restoration of the immune dysfunction to improve the patient survival.

\section{Acknowledgements}

The authors would like to thank Wirach Chitsuthipakorn for his technical advice. The authors wish to thank The $100^{\text {th }}$ Anniversary Chulalongkorn University for Doctoral Scholarship and The $90^{\text {th }}$ Anniversary of Chulalongkorn University Scholarship for the financial support.

\section{Conflict of interest}

(in the past 3 years)

Richard J Harvey is consultant with Medtronic, Stryker, Novartis, Meda, and NeilMed pharmaceuticals. Research grant funding received from Glaxo-Smith-Kline and Stallergenes. He has been on the speakers' bureau for Glaxo-SmithKline, Meda Pharmaceuticals and Seqirus.

Kornkiat Snidvongs received Honoraria for speaking at symposia from Merck Sharp \& Dohme, Bayer, AstraZeneca, and Menarini.

Thwe Phyo Kan Nyunt, Baharudin Abdullah, Maung Maung Khaing, Kachorn Seresirikachorn, Norasnieda Md Shukri, Songklot Aeumjaturapat, Jesada Kanjanaumporn and Supinda Chusakul report no conflicts of interest relevant to this article.

\section{Author contributions}

- Thwe Phyo Kan Nyunt: study design, data collection, data analysis, drafting the article, and final approval

- Baharudin Abdullah: data collection, manuscript edits

- Maung Maung Khaing: data collection, manuscript edits

- Kachorn Seresirikachorn: data collection, manuscript edits

- Norasnieda Md Shukri: data collection, manuscript edits

- Songklot Aeumjaturapat: data collection, manuscript edits

- Supinda Chusakul: data collection, manuscript edits

- Jesada Kanjanaumporn: data collection, manuscript edits

- Richard Harvey: expert opinion, data analysis, manuscript edits

- Kornkiat Snidvongs: conception, study design, data analysis, drafting the article, and final approval

\section{References}

1. Geerlings SE, Hoepelman AI. Immune dysfunction in patients with diabetes mellitus (DM). FEMS Immunol Med Microbiol. 1999;26(3-4): 259-65.

2. King GL. The role of inflammatory cytokines in diabetes and its complications. J Periodontol. 2008;79(8 Suppl):1527-34. 
3. Jafar N, Edriss H, Nugent K. The Effect of Short-Term Hyperglycemia on the Innate Immune System. Am J Med Sci. 2016;351:201-11.

4. Latgé JP. Aspergillus fumigatus and aspergillosis. Clin Microbiol Rev. 1999;12(2):310-50.

5. Petrikkos G, Skiada A, Lortholary O, Roilides E, Walsh TJ, Kontoyiannis DP. Epidemiology and Clinical Manifestations of Mucormycosis. Clin Infect Dis. 2012;54(Suppl 1):S23-S34.

6. Turner JH, Soudry E, Nayak JV, Hwang PH. Survival outcomes in acute invasive fungal sinusitis: a systematic review and quantitative synthesis of published evidence. Laryngoscope. 2013;123(5):1112-8.

7. Saedi B, Sadeghi M, Seilani P. Endoscopic management of rhinocerebral mucormycosis with topical and intravenous amphotericin B. J Laryngol Otol. 2011;125(8):807-10.

8. World Health Organization [Internet]. Geneva: World Health Organization; c2020 [cited 2020 Jun 8].; Definition and diagnosis of diabetes mellitus and intermediate hyperglycaemia: report of a WHO/ IDF consultation; [about 50 p.]. Available from: https://apps.who.int/iris/ handle/10665/43588

9. World Health Organization. Use of glycated haemoglobin (HbAlc) in diagnosis of diabetes mellitus: abbreviated report of a WHO consultation [Internet]. Geneva: World Health Organization; 2011 [cited 2020 Jun 8]. 25 p. Available from: https://apps.who.int/iris/handle/10665/7052310.

10. deShazo RD, O'Brien M, Chapin K, Soto-Aguilar M, Gardner L, Swain R. A new classification and diagnostic criteria for invasive fungal sinusitis. Arch Otolaryngol Head Neck Surg. 1997;123(11):1181-8.
11. Glassock RJ, Winearls C. Ageing and the glomerular filtration rate: truths and consequences. Trans Am Clin Climatol Assoc. 2009;120:419-28.

12. American Diabetes Association. Introduction: Standards of Medical Care in Diabetes 2020. Diabetes Care. 2020;43(Suppl1):S1-2.

13. Trachtenbarg DE. Diabetic ketoacidosis. Am Fam Physician. 2005;71(9): 1705-14.

14. Munker R. Leukocytosis, Leukopenia, and Other Reactive Changes of Myelopoiesis. In: Munker R, Hiller E, Glass J, Paquette R, editors. Modern Hematology: Biology and Clinical Management. Totowa, NJ: Humana Press; 2007. p. 127-35.

15. Asadollahi K, Hastings IM, Beeching NJ, Gill GV, Asadollahi P. Leukocytosis as an alarming sign for mortality in patients hospitalized in general wards. Iran J Med Sci. 2011;36(1):45-9.

16. Frater JL, Hall GS, Procop GW. Histologic features of zygomycosis: emphasis on perineural invasion and fungal morphology. Arch Pathol Lab Med. 2001;125(3):375-8.

17. Fokkens W, Lund V, Hopkins C, Hellings PW, Kern R, Reitsma S, et al. European Position Paper on Rhinosinusitis and Nasal Polyps 2020. Rhinology. 2020;58:1-464.

18. Sun HY, Forrest G, Gupta KL, Aguado JM, Lortholary O, Julia MB, et al Rhino-orbital-cerebral zygomycosis in solid organ transplant recipients. Transplantation. 2010;90(1):85-92.

19. Jung S-H, Kim SW, Park CS, Song CE, Cho JH, Lee JH, et al. Rhinocerebral Mucormycosis: Consideration of prognostic factors and treatment modality. Auris Nasus Larynx. 2009;36(3):274-9. 